
ПОЛИКИСТОЗА ЯИЧНИКОВ

\author{
(с) К.Д. Иевлева, И.Н. Данусевич, Л.В. Сутурина*
}

Научный центр проблем здоровья семьи и репродукции человека, Иркутск, Россия

Синдром поликистоза яичников (СПКЯ) является наиболее распространенной причиной эндокринного бесплодия у женщин. Одним из основных патогенетических звеньев данного заболевания является инсулинорезистентность. При этом механизмы развития СПКЯ при инсулинорезистентности окончательно не установлены. Лептин и рецептор, активируемый пролифераторами пероксисом гамма (Peroxisome Proliferator-Activated Receptor $\gamma ;$ PPARү), участвуют в регуляции углеводного обмена и репродуктивной функции, что указывает на возможность их вовлечения в патогенез СПКЯ. Статья представляет собой обзор публикаций по данной проблеме. Целью обзора явилась систематизация имеющихся сведений о молекулярных механизмах, определяющих роль лептина и РPARү в развитии СПкЯ. Литературный поиск проводился в период с 05.04.2020 по 17.05.2020 с использованием баз научной литературы: NCBI PubMed (зарубежные источники) и «Киберленинка» (отечественные источники), анализировались литературные источники за период 1990-2020 гг.

В обзоре отражены современные представления о роли лептина и PPARү в регуляции эндокринной, иммунной систем и репродуктивной функции, а также в развитии СПКЯ. Показано, что в настоящее время отсутствуют работы, освещающие механизмы взаимодействия лептина и PPARү в патогенезе данного синдрома. Имеющиеся публикации, указывающие на индивидуальный вклад в развитие заболевания и ассоциации лептина и РPARү с СПКЯ, представляют противоречивые результаты и обладают большим количеством ограничений. Это определяет актуальность дополнительного, более детального изучения прямого и непрямого взаимодействия лептина и РPARү в реализации физиологических процессов, а также в патогенезе СПкЯ.

КЛЮЧЕВЫЕ СЛОВА: СПКЯ; лептин; РРАRү; PРARG; ожирение; инсулинорезистентность; бесплодие.

\title{
ROLE OF LEPTIN AND NUCLEAR RECEPTOR PPARY IN PCOS PATHOGENESIS
}

๔ Kseniia D. levleva, Irina N. Danusivich, Larisa V. Suturina*

Scientific Centre for Family Health and Human Reproduction Problems, Irkutsk, Russia

Polycystic ovary syndrome (PCOS) is the most common cause of female endocrine infertility. Insulin resistance is supposed to be one of the essential factors of this disease pathways. At the same time, the mechanisms of PCOS development in insulin-resistant patients have not been completely established. Leptin and Peroxisome Proliferator-Activated Receptor $\gamma$ (PPAR $\gamma)$ are involved in carbohydrate metabolism and reproduction function regulation. It indicates that leptin and PPAR $y$ possibly play a role in the pathways of PCOS. This article is a review of publications on this issue. The purpose of this review was to systematize the available information on the molecular mechanisms that determine the role of leptin and PPAR $y$ in the development of PCOS. The literature search was carried out from 04/05/2020 to 05/17/2020 using the scientific literature databases: NCBI PubMed (foreign sources) and Cyberleninka (domestic sources). We analyzed publications for the period 1990-2020.

The review presents the current understanding of the possible role of leptin and PPAR $\gamma$ in the regulation of endocrine, immune systems, and reproductive function, as well as in the development of PCOS. Currently, no studies cover the mechanisms of interaction between leptin and PPARY in the pathways of this syndrome. The available studies indicating the individual contribution and association of leptin and PPAR $y$ with PCOS are conflicting and have many limitations. Therefore, more studies of direct and indirect interaction of leptin and PPARY, as well as their role in PCOS pathways, are needed.

KEYWORDS: PCOS; leptin; PPARY; PPARG; obesity; insulin resistance; infertility.

\section{ВВЕДЕНИЕ}

Синдром поликистоза яичников (СПКЯ) представляет собой состояние, которое характеризуется гирсутизмом и/или гиперандрогенемией, олигоановуляцией и/или поликистозной морфологией яичников и часто ассоциировано с клиническими проявлениями метаболического синдрома и повышенным риском развития сахарного диабета второго типа (СД2) [1-3].

Распространенность СПКЯ среди женщин репродуктивного возраста в некоторых популяциях достигает 19,9-21\% [4]. При этом рассматриваемое заболевание представляет собой наиболее частую причину бесплодия в данной возрастной группе [5]. 
Наряду с дисфункцией гипоталамо-гипофизарно-яичниковой оси значимым механизмом патогенеза СПКЯ считается гиперинсулинемия. Инсулинорезистентность и гиперинсулинемия встречаются у 65\% женщин с СПКЯ и ожирением и у $20 \%$ пациенток с СПКЯ и нормальным весом [6].

Известно, что ожирение, как и бесплодие, может развиваться в условиях снижения уровня или недостаточности функции гормона лептина. Лептин является главным медиатором передачи сигналов от жировой ткани и важным триггером для полового созревания и поддержания фертильности в целом, а также регулирует процессы пищевого поведения и энергетического метаболизма [7-9]. Часть из своих функций лептин реализует через рецептор, активируемый пролифераторами пероксисом гамма (Peroxisome Proliferator-Activated Receptor $\gamma$; PPARү), который регулирует множество метаболических, репродуктивных и иммунных процессов [10-12]. Нарушение работы данного рецептора может влиять на развитие инсулинорезистентности и ожирения, а инсулин, в свою очередь, регулирует экспрессию PPARY $[13,14]$.

Показана роль PPARү в регуляции выработки гипоталамических и овариальных гормонов, что, в свою очередь, указывает на его участие в функционировании репродуктивной системы [15]. Изменения в работе PPARY могут играть важную роль в патогенезе СПКЯ. Это подтверждается клиническими исследованиями с применением агонистов PPARү в качестве медикаментозной терапии у женщин, страдающих рассматриваемым заболеванием [16]. При этом изучены лишь некоторые молекулярные механизмы действия препаратов данной лекарственной группы $[17,18]$.

Целью данного обзора является систематизация имеющихся сведений о молекулярных механизмах, определяющих роль лептина и РPARү в развитии СПКЯ.

Литературный поиск проводился в период с 05.04.2020 по 17.05.2020 с использованием баз научной литературы: NCBI PubMed (зарубежные источники) и «Киберленинка» (отечественные источники), анализировались литературные источники за период 1990-2020 гг. Для поиска использовались следующие ключевые слова: СПКЯ, лептин, PPARy, PPARG, ожирение, инсулинорезистентность, бесплодие.

\section{ЛЕПТИН: ФИЗИОЛОГИЧЕСКАЯ РОЛЬ}

Лептин представляет собой пептидный гормон, который синтезируется преимущественно в жировой ткани, в результате чего его сывороточный уровень напрямую коррелирует с жировой массой. Так, снижение массы тела на $10 \%$ за счет сокращения количества жировой ткани приводит к снижению концентрации лептина в сыворотке крови на 53\% [19]. При этом увеличение массы тела на 10\% приводит к трехкратному увеличению концентрации лептина [20].

Рецепторы к лептину экспрессируются во многих тканях и органах, что обеспечивает его действие на всех уровнях гуморальной регуляции. Но основной эффект он оказывает, передавая сигналы в ядра медиобазального отдела гипоталамуса [21]. В результате этого происходит снижение аппетита и запускаются процессы, связанные с затратами энергии, которые в первую очередь направлены на метаболизм липидов и углеводов.

Так, лептин играет роль в регуляции углеводного обмена за счет увеличения чувствительности тканей к инсулину [21, 22]. Лептин стимулирует окисление липидов и синтез белков за счет супрессии липогенеза [22]. Этот механизм обусловлен элиминированием триглицеридов (ТГ) из жировой ткани без сопутствующего роста циркулирующих свободных жирных кислот (СЖК) посредством запуска митохондриального окисления [23]. Также имеются данные, что лептин коррелирует с индексом массы тела (ИМТ) как у человека, так и у лабораторных животных, независимо от массы и количества потребляемых калорий. В то же время окончательно не установлена природа вариабельности уровней лептина при сопоставимом ИМТ [24].

Исследования взаимодействия лептина и инсулина показывают, что уровень лептина коррелирует с уровнем инсулина. Количество вырабатывающегося лептина и инсулина напрямую зависит от массы жировой ткани, при этом избыток жировой массы тела ведет к появлению резистентности к данным гормонам, что в дальнейшем усугубляет состояние пациента, приводя к развитию таких заболеваний, как ожирение и СД2 [25, 26].

Помимо этого, имеются данные о прямом взаимодействии лептина и инсулина. Так, лептин, снижая глюконеогенез, а также синтез и секрецию инсулина, повышает чувствительность к нему различных тканей [27]. Инсулин, наоборот, стимулирует синтез и секрецию лептина $[28,29]$. Описанные процессы происходят как на уровне аутокринной [30], так и на уровне центральной регуляции [25]. Лептин и инсулин оказывают совместное действие на проопиомеланокортин-экспрессирующие нейроны (РОМС-нейроны), тем самым обеспечивая центральную регуляцию гомеостаза глюкозы [31].

Кроме того, лептин оказывает воздействие на репродуктивную систему. На уровне центральной регуляции лептин оказывает свой эффект за счет стимуляции выработки гонадотропин-рилизинг-гормона (GnRH) [32-34]. Также в реализацию центральных механизмов воздействия лептина вовлечены CART-нейроны (экспрессирующие кокаин и амфетамин-регулирующий пептид) гипоталамуса, участвующие в контроле пищевого поведения [35, 36]. На гипоталамо-гипофизарном уровне лептин способен напрямую стимулировать выработку лютеинизирующего гормона (ЛГ) и в меньшей мере - фолликулостимулирующего (ФСГ) через механизм активации NO-синтазы [37]. Рецепторы к лептину присутствуют в гранулезных клетках (ГК), клетках теки яичников и регулируются циклически. В результате лептин способен оказывать влияние на секрецию овариальных гормонов и действовать на созревание ооцитов и их потенциал [38, 39].

Лептин оказывает влияние на регуляцию как первичного, так и вторичного иммунного ответа через модуляцию пролиферации и активности клеток иммунной системы. Так, активация воспалительного ответа обеспечивается за счет стимулирования циклооксигеназы-2 (COX-2) и NO-синтазы, что также может играть роль в патогенезе многих заболеваний [40].

Таким образом, лептин, являясь гормоном жировой ткани и основным медиатором энергетического 
гомеостаза, вовлечен в функционирование и регуляцию различных процессов, в том числе углеводного метаболизма и репродуктивной функции. Вышеизложенное указывает на необходимость изучения его роли в патогенезе такого заболевания, как СПКЯ.

\section{PPARY: ФИЗИОЛОГИЧЕСКАЯ РОЛЬ}

Рецепторы, активируемые пролифераторами пероксисом (Peroxisome proliferator-activated receptor) (PPAR), относятся к семье ядерных рецепторов гормонов. Подсемейство PPAR состоит из альфа-, бетаи гамма-субтипов, которые контролируют экспрессию огромного количества генов, вовлеченных в метаболизм липидов и углеводов, энергообмен, адипогенез, воспаление и репродуктивную функцию [41]. Субтипы PPAR обладают различной физиологической активностью. Например, альфа- и бета-рецепторы участвуют в расходовании энергии, тогда как гамма-рецепторы стимулируют адипогенез [42]. Данные рецепторы экспрессируются практически во всех тканях организма, участвующих в метаболизме липидов: в печени, почках, кишечнике и скелетных мышцах [41, 42]. Изоформы PPAR обнаружены в яичниках многих видов животных, в том числе у человека [43]. Некоторые исследования указывают на важную роль PPAR в таких процессах в клетках яичников, как клеточный цикл, апоптоз и стероидогенез [44].

PPARY регулирует переключение метаболических процессов с внутриклеточного синтеза жирных кислот (ЖК) на поглощение ЖК адипоцитами и их депонирование в форме ТГ. Активация PPARү приводит к дифференцировке клеток жировой ткани и созреванию адипоцитов, способствуя ускорению процессов адипогенеза [45]. B то же время PPARү участвует в повышении чувствительности тканей к инсулину [13], стимуляции метаболизма глюкозы и липидов в печени и скелетных мышцах [46], подавлении продукции провоспалительных цитокинов в макрофагах [47]. В свою очередь, повышенное потребление жирной пищи ассоциировано с увеличением экспрессии PPARү в жировой ткани [11].

Некоторые исследования показывают прямое действие активации PPARү на клеточную утилизацию глюкозы. Данный механизм включает в себя активацию транспортера глюкозы 4 (GLUT4), стимуляцию сигнальных путей и фосфорилирование субстрата рецептора к инсулину (IRS-1) [48]. Активация PPAR также ассоциирована с положительным воздействием на адипонектин, резистин, интерлейкин-6 (ИЛ-6), фактор некроза опухоли (ФНО), продукцию СЖК и другими механизмами, что, в частности, подтверждается результатами экспериментов с агонистами PPAR $[49,50]$. Таким образом, PPAR является одним из основных внутриклеточных регуляторов чувствительности к инсулину.

Установлено, что PPARү играет важную роль в репродуктивной функции. В яичниках он влияет на созревание ооцитов, регуляцию овуляции и регрессию желтого тела [51], контролирует овариальный стероидогенез, стимулирует секрецию прогестерона [43]. PPARү детектируется в клетках фолликулов в фазе раннего фолликулогенеза, и его уровень увеличивается до преовуляторной фазы, затем снижаясь под действием нарастающего
ЛГ $[15,51]$. Использование мутантной мышиной модели с делецией PPARG в гранулезных клетках привело к нарушению овуляции вследствие дефекта разрыва фолликулов [51]. При этом динамика изменений PPARү отличается среди разных видов, и для человеческого организма исследована не полностью $[10,15]$.

С другой стороны, показано, что экспрессия PPARү регулируется различными гормонами и факторами. В фолликулах яичников гормон резистин увеличивает экспрессию PPARү [52], а в гранулезной ткани крыс наблюдается снижение его экспрессии после пика концентрации ЛГ [51]. В исследованиях на обезьянах показано, что ЛГ и хорионический гонадотропин (ХГЧ) быстро снижают экспрессию PPAR в преовуляторных фолликулах [12], при этом уровень данного рецептора повышается под действием ФСГ, прогестерона, эстрадиола, но не тестостерона [43]. PPARү играет важную роль в иммунном ответе через ингибирование экспрессии провоспалительных цитокинов (ФНО и ИЛ-6) и напрямую через стимуляцию дифференцировки клеток иммунного ответа в направлении антивоспалительного фенотипа [10]. В свою очередь, ФНО и ИЛ-6 положительно коррелируют с уровнем глюкозы натощак и наличием инсулинорезистентности у женщин с СПКЯ [53]. Показано, что система PPARY регулирует синтез простагландинов макрофагами через модуляцию экспрессии СОХ-2 - одного из главных ферментов данного процесса [54]. При этом у мышей с делецией данного гена наблюдается сниженная репродуктивная функция [55].

Таким образом, PPARү оказывает системное влияние на метаболизм жиров и углеводов, регуляцию воспалительного процесса и играет важную роль в функционировании репродуктивной системы, что косвенно указывает на его участие в патогенезе СПкЯ.

\section{ВЗАИМОДЕЙСТВИЕ ЛЕПТИНА И РРАRҮ НА РАЗНЫХ УРОВНЯХ РЕГУЛЯЦИИ}

Несмотря на достаточное количество клинических исследований, показывающих наличие взаимосвязи между лептином и PPARү, в настоящее время отсутствуют ясные представления о механизмах их взаимодействия, в особенности на уровне репродуктивной системы. Показано, что транскрипционная активность гена лептина снижается в присутствии лигандов, активирующих PPARy [56]. На уровне центральной нервной системы изменение экспрессии PPARү в POMC-нейронах влияет на их чувствительность к лептину, что подтверждается в экспериментах на линии мышей с делецией PPARү в POMC-нейронах [57]. На основе этих данных можно предположить, что повышенная экспрессия PPARү может стать одной из причин развития лептинорезистентности. С другой стороны, обнаружено, что лептин через свои центральные механизмы подавляет экспрессию PPARү в жировой ткани [58]. В то же время непосредственно на уровне жировой ткани у крыс под действием введения лептина экспрессия PPAR $ү$ повышается [59]. Однако в исследованиях на мышах с делецией гена лептина его введение не влияло на экспрессию PPARY [60].

Многие регуляторные процессы в адипоцитах, находящиеся под контролем PPARү, в частности, контроль 
за продукцией неэстерифицированных СЖК, влияют и на функцию тканей яичников. В результате любая активация PPARү в жировой ткани способна воздействовать на репродуктивный потенциал [61,62].

Таким образом, существующие данные о взаимовлиянии лептина и PPARү указывают на их совместное участие в метаболических процессах и репродуктивной функции, но не раскрывают точные механизмы этого взаимодействия в органах и тканях. Это объясняется влиянием множества дополнительных факторов и процессов, которые также играют роль в регуляции лептина и PPAR $\gamma$, в связи с чем определить изолированный эффект этих двух компонентов метаболических процессов представляет из себя сложную задачу.

\section{РОЛЬ ЛЕПТИНА И РРАRY В ПАТОГЕНЕЗЕ СПКЯ}

Оценке изолированного участия лептина и PPARY в патогенезе различных заболеваний, в том числе СПКЯ, посвящено достаточно большое количество исследований

Известно, что избыточная масса тела и ожирение характерны для женщин, страдающих СПКЯ [63]. При этом при СПКЯ наблюдается связь концентрации лептина в сыворотке крови со значениями ТГ, инсулина, грелина, уровнем секреции инсулина и наличием инсулинорезистентности [64]. Как обсуждалось выше, имеются свидетельства того, что инсулин стимулирует синтез и секрецию лептина $[28,29]$. Однако механизмы этого воздействия окончательно не установлены. По-видимому, ключевую роль в регуляции экспрессии МРНК лептина играет инсулино-стимулированный метаболизм глюкозы [29]. Так, согласно данным Vidal (1996), инсулин не повышает синтез мРНК лептина в адипоцитах, хотя вызывает значительное увеличение мPHK Glut 4 [65].

В эксперименте показано, что половые стероиды контролируют уровень лептина [66], а ГК способны синтезировать и запасать лептин с рождения независимо от объема жировой ткани [67]. Установлено, что гиперлептинемия и высокий уровень лептина в фолликулярной жидкости (ФЖ) могут влиять на стероидогенез, репродуктивную функцию и фертильность [68]. Интересно, что в сыворотке крови и в ФЖ у женщин с СПКЯ зарегистрирован высокий уровень лептина в сравнении с аналогичными показателями у женщин контрольной группы [69]. Также показано, что у женщин с СПКЯ и избыточной массой тела концентрация сывороточного лептина и отношения лептин/адипонектин значимо выше, чем их значения при СПКЯ и нормальной массе тела [70]. В то же время в когорте женщин с СПКЯ и нормальной массой тела наблюдался значимо более низкий уровень лептина в ФЖ в сравнении с овулирующими женщинами и положительная корреляция между его уровнем в сыворотке крови и инсулинемией [7].

Как описано выше, PPARү является одним из внутриядерных регуляторов чувствительности клеток к инсулину. В исследовании Qu F. et al. [71] продемонстрировано ингибирование PPARү под воздействием андрогенов у женщин, страдающих СПКЯ. С другой стороны, применение агонистов PPAR $ү$ приводит к ингибированию ЛГ и инсулин-стимулированного синтеза андрогенов, что снижает уровень тестостерона у пациенток с СПКЯ.
Отмечено воздействие препаратов тиазолидонового ряда и на уровень эстрогенов, однако их эффекты зависят от возраста и эндокринного статуса пациенток [72]. Некоторыми исследователями обнаружено снижение экспрессии PPARү в ГК яичников у женщин с СПКЯ [73], хотя другие авторы описывают ее повышение в яичниках [74]. По мнению M.Vitti (2016), данные об экспрессии и функции PPAR при заболеваниях, связанных с бесплодием, ограничены, и этот вопрос заслуживает дальнейшего изучения [75].

Известно, что уровень PPARү ассоциирован с COX-2 [73] и обеспечивает контроль экспрессии генов провоспалительных цитокинов, а именно ИЛ-6 и ФНО [76]. У женщин, страдающих бесплодием на фоне СПКЯ, обнаружены высокие уровни сывороточных и фолликулярных концентраций ФНО и ИЛ-6 [77], а применение агонистов PPARү при лечении СПКЯ приводит к снижению выработки провоспалительных цитокинов, чем объясняется улучшение воспалительного статуса пациенток [78]. Таким образом, низкий уровень экспрессии PPARү с нарушением равновесия между про- и противовоспалительными цитокинами может рассматриваться как важное звено патогенеза СПКЯ

До настоящего времени практически отсутствуют исследования, описывающие характер взаимодействия лептина и PPARү у женщин, страдающих СПКЯ. Ряд работ посвящен изучению роли полиморфизмов их генов в развитии различных метаболических нарушений $[79,80]$. В метаанализе 33 исследований взаимодействия полиморфизмов генов рецептора лептина (LEPR) и PPAR (PPARG) установлено, что носительство полиморфизма LEPR rs1137101 и полиморфизмов PPARG rs1801282 и rs3856806 ассоциировано с СПКЯ [81], при этом до сих пор отсутствуют данные о межгенных взаимодействиях данных полиморфных вариантов.

Таким образом, несмотря на установленную роль лептина и PPARү в репродуктивной функции, а также наличие исследований, демонстрирующих их возможное влияние друг на друга, остается нерешенным вопрос об их взаимном участии в патогенезе СПКя. Для решения данного вопроса необходимо детально изучить физиологическую роль взаимодействия лептина и PPAR $\gamma$, что позволит планировать исследования с учетом всех установленных ассоциаций и механизмов.

\section{ЗАКЛЮЧЕНИЕ}

Лептин и PPARү играют важную роль в эндокринной, иммунной и репродуктивной регуляции, а также в развитии СПКЯ. Однако данных, освещающих механизмы их взаимодействия в патогенезе данного заболевания, как и исследований, раскрывающих их взаимовлияние в реализации физиологических функций, недостаточно.

С каждым годом увеличивается количество исследований, указывающих на индивидуальный вклад лептина и PPARү в патогенез СПКЯ, в то же время имеющиеся работы представляют противоречивые результаты и обладают большим количеством ограничений. Это определяет актуальность дополнительного, более детального, изучения прямого и непрямого взаимодействия лептина и PPARү в реализации физиологических процессов, а также в патогенезе СПКЯ. 


\section{ДОПОЛНИТЕЛЬНАЯ ИНФОРМАЦИЯ}

Источник финансирования. Работа выполнена по инициативе авторов без привлечения финансирования.

Конфликт интересов. Авторы заявляют об отсутствии конфликта интересов.

Информация о вкладе каждого автора: Иевлева К.Д. - лите- ратурный поиск и анализ, написание текста статьи; Данусевич И.Н. написание текста статьи; Сутурина Л.В. - общее редактирование текста статьи.

Все авторы одобрили финальную версию статьи перед публикацией, выразили согласие нести ответственность за все аспекты работы, подразумевающую надлежащее изучение и решение вопросов, связанных с точностью или добросовестностью любой части работы.

\section{СПИСОК ЛИТЕРАТУРЫ | REFERENCES}

1. Fauser BC, Tarlatzis BC, Rebar RW, et al. Consensus on women's health aspects of polycystic ovary syndrome (PCOS): the Amsterdam ESHRE/ASRM-Sponsored 3rd PCOS Consensus Workshop Group. Fertil Steril. 2012;97(1):28-38.e25. doi: https://doi.org/10.1016/j. fertnstert.2011.09.024 Epub 2011 Dec 6. PMID: 22153789.

2. Lizneva D, Kirubakaran R, Mykhalchenko K, Suturina L, et al. Phenotypes and body mass in women with polycystic ovary syndrome identified in referral versus unselected populations: systematic review and meta-analysis. Fertil Steril. 2016;106(6):1510-1520.e2. doi: https://doi.org/10.1016/j.fertnstert.2016.07.1121

3. Suturina LV, Atalyan AV, Darzhaev ZY, et al. Overweight and obesity prevalence in referral population of infertile women with polycystic ovary syndrome. Advances in Obesity, Weight Management and Control. 2017; 7(1):00188. doi: https://doi.org/10.15406/aowmc.2017.07.00188

4. Belenkaia LV, Lazareva LM, Walker W, et al. Criteria, phenotypes and prevalence of polycystic ovary syndrome. Minerva Ginecol. 2019; 71(3):211-223. doi: https://doi.org/10.23736/S0026-4784.19.04404-6

5. Deshpande P, Gupta A. Causes and prevalence of factors causing infertility in a public health facility. J Hum Reprod Sci. 2019; 12(4):287. doi: https://doi.org/10.4103/jhrs.JHRS_140_18

6. Dale PO, Tanbo T., Vaaler S., Abyholm T. Body weight, hyperinsulinemia, and gonadotropin levels in the polycystic ovarian syndrome: evidence of two distinct populations. Fertility and Sterility. 1992; 58(3):487-491. doi: https://doi.org/10.1016/s0015-0282(16)55249-2.

7. Garruti G, de Palo R, Rotelli MT, et al. Association between Follicular Fluid Leptin and Serum Insulin Levels in Nonoverweight Women with Polycystic Ovary Syndrome. Biomed Res Int. 2014;2014:1-7. doi: https://doi.org/10.1155/2014/980429.

8. Seufert J. Leptin Effects on Pancreatic $\beta$-Cell Gene Expression and Function. Diabetes. 2004;53(Supplement 1):S152-S158. doi: https://doi.org/10.2337/diabetes.53.2007.S152.

9. Spicer LS, Francisco CC. The adipose obese gene product, leptin: evidence of a direct inhibitory role in ovarian function. Endocrinology. 1997;138(8):3374-9. doi: https://doi.org/10.1210/endo.138.8.5311.

10. Minge CE, Robker RL, Norman RJ. PPAR gamma: coordinating metabolic and immune contributions to female fertility. Publishing Corporation PPAR Research. 2008;2008:243791. doi: https://doi.org/10.1155/2008/243791

11. Nicol CJ, Adachi M., Akiyama TE, Gonzalez FJ. PPAR gamma in endothelial cells influences high fat dietinduced hypertension. Am J Hypertens. 2005;4(1):549-56. doi: https://doi.org/10.1016/j.amjhyper.2004.10.032.

12. Puttabyatappa M, Vandevoort CA, Chaffin CL. HCG induced downregulation of PPARg and liver $X$ receptors promotes periovulatory progesterone synthesis by macaque granulosa cells. Endocrinology. 2010;151:5865-5872. doi: https://doi.org/10.1210/en.2010-0698

13. Hevener AL, Olefsky JM, Reichart D, et al. Macrophage PPAR gamma is required for normal skeletal muscle and hepatic insulin sensitivity and full antidiabetic effects of thiazolidinediones. J. Clin. Invest. 2007;117:1658-1669. doi: https://doi.org/10.1172/JCl31561

14. Rieusset J, Andreelli F, Auboeuf D, et al. Insulin acutely regulates the expression of the peroxisome proliferator-activated receptorgamma in human adipocytes. Diabetes. 1999;48:699-705. doi: https://doi.org/10.2337/diabetes.48.4.699.

15. Froment P, Gizard F, Defever D, et al. Peroxisome proliferatoractivated receptors in reproductive tissues: from gametogenesis to parturition. J Endocrinol. 2006;189:199-209. doi: https://doi.org/10.1677/joe.1.06667.

16. Du Q, Yang S, Wang Y-J, et al. Effects of thiazolidinediones on polycystic ovary syndrome: a meta-analysis of randomized placebo-controlled trials. Adv Ther. 2012;29(9):763-74. doi: https://doi.org/10.1007/s12325-012-0044-6.
17. Seto-Young D, Paliou M, Schlosser J, et al. Direct Thiazolidinedione action in the human ovary: insulinindependent and insulinsensitizing effects on steroidogenesis and insulin-like growth factor binding protein-1 production. J Clin Endocrinol Metab. 2005;90:6099-6105. doi: https://doi.org/10.1210/jc.2005-0469.

18. Minge CE, Ryan NK, Van Der Hoek KH, et al. Troglitazone regulates peroxisome proliferator-activated receptors and inducible nitric oxide synthase in murine ovarian macrophages. Biol Reprod. 2006;74:153-160. doi: https://doi.org/10.1095/biolreprod

19. Considine R V., Sinha MK, Heiman ML, et al. Serum Immunoreactive-Leptin Concentrations in Normal-Weight and Obese Humans. N Engl J Med. 1996;334(5):292-295. doi: https://doi.org/10.1056/NEJM199602013340503

20. Kolaczynski JW, Ohannesian JP, Considine RV, et al. Response of leptin to short-term and prolonged overfeeding in humans. J. Clin. Endo. Metab. 1996;81(11):4162-4165. doi: https://doi.org/10.1210/jcem.81.11.8923877.

21. Morton GJ, Gelling RW, Niswender KD, Morrison CD, Rhodes CJ, Schwartz MW. Leptin regulates insulin sensitivity via phosphatidylinositol-3-OH kinase signaling in mediobasal hypothalamic neurons. Cell Metab. 2005;2(6):411-420. doi: https://doi.org/10.1016/j.cmet.2005.10.009

22. Barzilai N, Wang J, Massilon D, et al. Leptin selectively decreases visceral adiposity and enhances insulin action. J Clin Invest. 1997;100:3105-3110. doi: https://doi.org/10.1172/JCI119865

23. Shimabukuro M, Koyama K, Chen G, et al. Direct antidiabetic effect of leptin through triglyceride depletion of tissues. Proc Natl Acad SCi USA. 1997;94:4637-4641. doi: https://doi.org/10.1073/pnas.94.9.4637

24. Maffei M, Halaas J, Ravussin E., et al. Leptin levels in human and rodent: measurement of plasma leptin and ob RNA in obese and weight-reduced subjects. Nat Med. 1995;1(11):1155-61. doi: https://doi.org/10.1038/nm1195-1155

25. Marino JS, Xu Y, Hill JW. Central insulin and leptin-mediated autonomic control of glucose homeostasis. Trends Endocrinol Metab. 2011;37(9):2053-2079. doi: https://doi.org/10.1016/j.tem.2011.03.001

26. Ceddia RB, Koistinen HA, Zierath JR. Analysis of paradoxical observations on the association between leptin and insulin resistance. Sweeney GFASEB J. 2002;16(10):1163-76. doi: https://doi.org/10.1096/fj.02-0158rev

27. Park S, Ahn IS, Kim DS. Central infusion of leptin improves insulin resistance and suppresses beta-cell function, but not betacell mass, primarily through the sympathetic nervous system in a type 2 diabetic rat model. Life Sci. 2010;86(23-24):854-62. doi: https://doi.org/10.1016/j.lfs.2010.03.021

28. Laferrere B, Caixas A, Fried S, Bashore C, et al. A pulse of insulin and dexamethasone stimulates serum leptin in fasting human subjects. Eur J Endocrinol. 2002:839-845. doi: https://doi.org/10.1530/eje.0.1460839

29. Moreno-Aliaga MJ, Stanhope KL, Havel PJ. Transcriptional regulation of the leptin promoter by insulin-stimulated glucose metabolism in 3t3-I1 adipocytes. Biochem Biophys Res Commun. 2001;283:544-548. doi: https://doi.org/10.1006/bbrc.2001.4822.

30. Müller G, Ertl J, Gerl M, Preibisch G. Leptin impairs metabolic actions of Insulin in isolated rat adipocytes. J Biol Chem. 1997;272(16):10585-93. doi: https://doi.org/10.1074/jbc.272.16.10585.

31. Hill JW, Elias CF, Fukuda M, et al. Direct insulin and leptin action on pro-opiomelanocortin neurons is required for normal glucose homeostasis and fertility. Cell Metab. 2010;11(4):286-97. doi: https://doi.org/10.1016/j.cmet.2010.03.002

32. Lebrethon MC, Vandersmissen E, Gerard A, et al. In vitro stimulation of the prepubertal rat gonadotropin-releasing hormone pulse generator by leptin and neuropeptide $Y$ through distinct mechanisms. Endocrinology. 2000;141:1464-9. doi: https://doi.org/10.1210/endo.141.4.7432. 
33. Cunningham MJ, Clifton DK, Steiner RA. Leptin's actions on the reproductive axis: perspectives and mechanisms. Biol Reprod. 1999;60:216-22. doi: https://doi.org/10.1095/biolreprod60.2.216.

34. Magni P, Vettor R, Pagano C, et al. Expression of a leptin receptor in immortalized gonadotropin-releasing hormonesecreting neurons. Endocrinology. 1999;140:1581-5. doi: https://doi.org/10.1210/endo.140.4.6622

35. Parent AS, Lebrethon MC, Gerard A, et al. Leptin effects on pulsatile gonadotropin releasing hormone secretion from the adult rat hypothalamus and interaction with cocaine and amphetamine regulated transcript peptide and neuropeptide. Regul Pept. 2000;92:17-24. doi: https://doi.org/10.1016/S0167-0115(00)00144-0

36. Lebrethon MC, Vandersmissen E, Gerard A, et al. Cocaine and amphetamine-regulated-transcript peptide mediation of leptin stimulatory effect on the rat gonadotropin-releasing hormone pulse generator in vitro. J Neuroendocrinol. 2000;12:383-5. doi: https://doi.org/10.1046/j.13652826.2000.00497.x.

37. YuWH, Kimura M, Walczewska A, et al. Role of leptin in hypothalamicpituitary function. Proc Natl Acad Sci USA. 1997;94:1023-8. doi: https://doi.org/10.1073/pnas.94.3.1023.

38. Ryan NK, Woodhouse CM, Van der Hoek KH, et al. Expression of Leptin and Its Receptor in the Murine Ovary: Possible Role in the Regulation of Oocyte Maturation 1. Biol Reprod. 2002;66(5):1548-1554. doi: https://doi.org/10.1095/biolreprod66.5.1548

39. Zachow RJ, Magoffin MD. Direct Intraovarian Effects of Leptin: Impairment of the Synergistic Action of InsulinLike Growth factor-I on Follicle-Stimulating HormoneDependent estradiol-17 Beta Production by Rat Ovarian Granulosa Cells. Endocrinology. 1997;138(2):847-50. doi: https://doi.org/10.1210/endo.138.2.5035

40. Becerril S, Rodríguez A, Catalán V, et al. Functional Relationship between Leptin and Nitric Oxide in Metabolism. Nutrients. 2019;11(9):2129. doi: https://doi.org/10.3390/nu11092129

41. Fajas L, Debril MB, Auwerx J. Peroxisome proliferatoractivated receptor-g: from adipogenesis to carcinogenesis. J Mol Endocrinol. 2001;27:1-9. doi: https://doi.org/10.1677/jme.0.0270001.

42. Tyagi S, Gupta P, Singh A, et al. The peroxisome proliferatoractivated receptor: A family of nuclear receptors role in various diseases. J Adv Pharm Technol Res. 2011;2:236-240. doi: https://doi.org/10.4103/2231-4040.90879

43. Kurowska P, Chmielinska J, Ptak A, Rak A. Expression of peroxisome proliferator-activated receptors is regulated by gonadotropins and steroid hormones in in vitro porcine ovarian follicles. Journal of Physiology and Pharmacology. 2017;68(6):823-832.

44. Komar CM. Peroxisome proliferator-activated receptors (PPARs) and ovarian function: implications for regulating steroidogenesis, differentiation, and tissue remodeling. Reprod Biol Endocrinol. 2005:3:41. doi: https://doi.org/10.1186/1477-7827-3-41

45. Walczak R, Tontonoz P. PPARadigms and PPARadoxes: Expanding roles for PPARy in the control of lipid metabolism. J Lipid Res. 2002;43:177-186 doi: https://doi.org/10.1016/S0022-2275(20)30159-0.

46. Gavrilova O, Haluzik M, Matsusue K., et al. Liver peroxisome proliferatoractivated receptor gamma contributes to hepatic steatosis, triglyceride clearance, and regulation of body fat mass. Biol Chem. 2003;278(36):34268-76. doi: https://doi.org/10.1074/jbc.M300043200

47. Vats D, Mukundan L, Odegaard JI, et al. Oxidative metabolism and PGC-1 beta attenuate macrophagemediated inflammation. Cell Metab. 2006:4:13-24. doi: https://doi.org/10.1016/j.cmet.2006.05.011.

48. Armoni M, Kritz N, Harel C, et al. Peroxisome proliferatoractivated receptor-gamma represses GLUT4 promoter activity in primary adipocytes, and rosiglitazone alleviates this effect. J Biol Chem. 2003;15;278(33):30614-23. doi: https://doi.org/10.1074/jbc.M304654200

49. Sharma AM, Staels B. Review: Peroxisome proliferator-activated receptor gamma and adipose tissue - understanding obesity-related changes in regulation of lipid and glucose metabolism. J Clin Endocrinol Metab. 2007;92:386-395. doi: https://doi.org/10.1210/jc.2006-1268.

50. Okuno A, Tamemoto $\mathrm{H}$, Tobe $\mathrm{K}$, et al. Troglitazone increases the number of small adipocytes without the change of white adipose tissue mass in obese Zucker rats. J Clin Invest. 1998;101:1354-1361. doi: https://doi.org/10.1172/JCl1235.

51. Komar CM, Braissant O, Wahli W, Curry TE. Expression and localization of PPARs in the rat ovary during follicular development and the periovulatory period. Endocrinology. 2001;142:4831-4838. doi: https://doi.org/10.1210/endo.142.11.8429.

52. Rak-Mardyla A, Drwal E. In vitro interaction between resistin and peroxisome proliferator-activated receptor $\mathrm{g}$ in porcine ovarian follicles. Reprod Fertil Dev. 2016; 28(3):357-368. doi: https://doi.org/10.1071/RD14053

53. Tarkun I, Cetinarslan B, Türemen E, et al. Association between Circulating Tumor Necrosis Factor-Alpha, Interleukin-6, and Insulin Resistance in Normal-Weight Women with Polycystic Ovary Syndrome. Metab Syndr Relat Disord. 2006;4(2):122-128. doi: https://doi.org/10.1089/met.2006.4.122

54. Subbaramaiah K, Lin DT, Hart JC, Dannenberg AJ. Peroxisome proliferator-activated receptor gamma ligands suppress the transcriptional activation of cyclooxygenase-2: evidence for involvement of activator protein-1 and CREBbinding protein/ p300. J Biol Chem. 2001;276:12440-12448. doi: https://doi.org/10.1074/jbc.W119.012136

55. Lim H, Paria BC, Das SK, et al. Multiple female reproductive failures in cyclooxygenase 2-deficient mice. Cell. 1997;91:197-208. doi: https://doi.org/10.1016/s0092-8674(00)80402-x

56. De Vos P, Lefebvre AM, Miller SG, et al. Thiazolidinediones repress ob gene expression in rodents via activation of peroxisome proliferator-activated receptor gamma. J Clin Invest. 1996;98(4):1004-1009. doi: https://doi.org/10.1172/JCI118860.

57. Long L, Toda C, et al. PPARy ablation sensitizes proopiomelanocortin neurons to leptin during high-fat feeding. J Clin Invest. 2014;124(9):4017-4027. doi: https://doi.org/10.1172/JCI76220

58. Buettner C, Muse ED, Cheng A, et al. Leptin controls adipose tissue lipogenesis via central, STAT3-independent mechanisms. Nat Med. 2008;14(6):667-675. doi: https://doi.org/10.1038/nm1775.

59. Qian H, Hausman GJ, Compton MM, et al. Leptin Regulation of Peroxisome Proliferator-Activated Receptor-Gamma, Tumor Necrosis Factor, and Uncoupling protein-2 Expression in Adipose Tissues. Biochem Biophys Res Commun. 1998;246(3):660-7. doi: https://doi.org/10.1006/bbrc.1998.8680.

60. Zhang W, Della-Fera MA, Hartzell DL, et al. Adipose tissue gene expression profiles in ob/ob mice treated with leptin. Life Sciences. 2008;83(2008):35-42. doi: https://doi.org/10.1016/j.lfs.2008.04.021

61. Mitchell M, Armstrong DT, Robker RL, Norman RJ. Adipokines: implications for female fertility and obesity. Reproduction. 2005;130(5):583-597. doi: https://doi.org/10.1530/rep.1.00521

62. Mu YM, Yanase T, Nishi Y, et al. Insulin sensitizer, troglitazone, directly inhibits aromatase activity in human ovarian granulosa cells. Biochem Biophys Res Commun. 2000; 271:710-713. doi: https://doi.org/10.1006/bbrc.2000.2701.

63. Pasquali R, Gambineri A. Polycystic ovary syndrome: a multifaceted disease from adolescence to adult age. Annals of the New York Academy of Sciences. 2006;1092:158-174. doi: https://doi.org/10.1196/annals.1365.014.

64. Daghestani MH, Daghestani M, Daghistani M, et al. Study of ghrelin and leptin levels and their relationship to metabolic profiles in obese and lean Saudi women with polycystic ovary syndrome (PCOS). Lipids Health Dis. 2018;17:195. doi: https://doi.org/10.1186/s12944-018-0839-9

65. Vidal H, Auboeuf D, De Vos P, et al. The expression of ob gene is not acutely regulated by Insulin and fasting in human abdominal subcutaneous adipose tissue. J Clin Invest. 1996;98(2):251-255. doi: https://doi.org/10.1172/JCI118786.

66. Pinilla L, Seoane L, Gonzalez L, et al. Regulation of serum leptin levels by gonadal function in rats. Eur J Endocrinol. 1999:468-473. doi: https://doi.org/10.1530/eje.0.1400468

67. Abir R, Ao A, Jin S, et al. Leptin and its receptors in human fetal and adult ovaries. Fertility and Sterility. 2005; 84 (6):1779-1782. doi: https://doi.org/10.1016/j.fertnstert.2005.05.067

68. De Placido G, Alviggi C, Clarizia R, et al. Intra-follicular leptin concentration as a predictive factor for in vitro oocyte fertilization in assisted reproductive techniques. Journal of Endocrinological Investigation. 2006; 29(8):719-726. doi: https://doi.org/10.1007/BF03344182.

69. Li M-G, Ding G-L, Chen X-J, et al. Association of serum and follicular fluid leptin concentrations with granulosa cell phosphorylated signal transducer and activator of transcription 3 expression in fertile patients with polycystic ovarian syndrome. Journal of Clinical Endocrinology and Metabolism. 2007;92(12):4771-4776. doi: https://doi.org/10.1210/jc.2007-0978. 
70. Savastano S, Valentino R, Di Somma C, et al. Serum 25- Hydroxyvitamin D Levels, phosphoprotein enriched in diabetes gene product (PED/PEA15 ) and leptin-to-adiponectin ratio in women with PCOS. Nutrition and Metabolism. 2011;8:84. doi: https://doi.org/10.1186/1743-7075-8-84.

71. Qu F, Wang FF, Yin R, et al. A molecular mechanism underlying ovarian dysfunction of polycystic ovary syndrome: hyperandrogenism induces epigenetic alterations in the granulosa cells. J Mol Med (Berl). 2012;90:911-923. doi: https://doi.org/10.1007/s00109-012-0881-4

72. Froment $P$, Touraine P. Thiazolidinediones and Fertility in Polycystic Ovary Syndrome (PCOS). PPAR Res. 2006;2006:73986. doi: https://doi.org/10.1155/PPAR/2006/73986

73. Lee JY, Tae JC, Kim CH, et al. Expression of the genes for peroxisome proliferator activated receptor- $\gamma$, cyclooxygenase-2, and proinflammatory cytokines in granulosa cells from women with polycystic ovary syndrome. Clin Exp Reprod Med. 2017;44(3):146-151. doi: https://doi.org/10.5653/cerm.2017.44.3.146.

74. Jansen E, Laven JS, Dommerholt HB, et al. Abnormal gene expression profiles in human ovaries from polycystic ovary syndrome patients. Mol Endocrinol. 2004;18:3050-3063. doi: https://doi.org/10.1210/me.2004-0074

75. Vitti M, Di Emidio G, Di Carlo M, et al. Peroxisome ProliferatorActivated Receptors in Female Reproduction and Fertility. PPAR Res. 2016;2016:1-12. doi: https://doi.org/ 10.1155/2016/4612306

76. Jiang C, Ting AT, Seed B. PPAR- $y$ agonists inhibit production of monocyte inflammatory cytokines. Nature. 1998;391(6662):82-86. doi: https://doi.org/10.1038/34184.
77. Al-Musawy SHH, Al-Saimary IE, Flaifil MS Levels of cytokines profile in polycystic ovary syndrome. Medical Journal of Babilon. 2018; 15(2):124-128. doi: https://doi.org/10.4103/MJBL.MJBL_32_18.

78. Guo Q, Shan J, Xu Y, et al. Pioglitazone Metformin Complex Improves Polycystic Ovary Syndrome Comorbid Psychological Distress via Inhibiting NLRP3 Inflammasome Activation: A Prospective Clinical Study. Mediators Inflamm. 2020;2020:1-7. doi: https://doi.org/10.1155/2020/3050487.

79. Sheneman E, Bairova T, levleva K, et al. GP222 Clinical and metabolic parameters in girls-carriers of LEPR rs1 137100 with android and gynoid obesity. In: Abstracts. BMJ Publishing Group Ltd and Royal College of Paediatrics and Child Health; 2019:A120.3-A121 doi: https://doi.org/10.1136/archdischild-2019-epa.281.

80. Иевлева К.Д., Баирова Т.А., Шенеман Е.А., и др. Протективный эффект G-аллеля полиморфизма PPARG2 rs 1801282 в отношении избыточной массы тела и ожирения у подростков-монголоидов // Журнал медико-биологических исследований. — 2019. - Т. 7. — №4. - С. 452-463. [levleva KD, Bairova TA, Sheneman EA, et al. The protective effect of G-allele of PPARG2 rs1801282 polymorphism against overweight and obesity in mongoloid adolescents. Journal of Medical and Biological Research. 2019;7(4):452-463. (In Russ.)]. doi: https://doi.org/10.17238/issn2542-1298.2019.7.4.452

81. Liang J, Lan J, Li M, Wang F. Associations of leptin receptor and peroxisome proliferator-activated receptor gamma polymorphisms with polycystic ovary syndrome: a meta-analysis. Ann Nutr Metab. 2019; 75(1):1-8. doi: https://doi.org/10.1159/000500996.

Рукопись получена: 02.09.2020. Одобрена к публикации: 06.12.2020. Опубликована online: 30.12.2020.

\section{ИНФОРМАЦИЯ ОБ АВТОРАХ [AUTHORS INFO]}

*Сутурина Лариса Викторовна, профессор, д.м.н. [Larisa V. Suturina, MD, PhD]; 664003, ул. Тимирязева, д. 16, Иркутск, Россия [Timiryzeva st. 16, Irkutsk, Russia, 664003]; ORCID: https://orcid.org/0000-0002-6271-7803X; eLibrary SPIN: 9419-0244; e-mail: Isuturina@mail.ru

Иевлева Ксения Дмитриевна [Kseniia D. levleva]; ORCID: https://orcid.org/0000-0002-0177-234X; eLibrary SPIN: 2743-9932; e-mail: asiy91@mail.ru

Данусевич Ирина Николаевна, А.М.H. [Irina N. Danusivich, MD, PhD]; ORCID: https://orcid.org/0000-0002-8862-5771X; eLibrary SPIN: 6289-3358; e-mail: irinaemails@gmail.com

\section{ЦИТИРОВАТЬ:}

Иевлева К.Д., Данусевич И.Н., Сутурина Л.В. Роль лептина и ядерного рецептора PPARү в патогенезе синдрома поликистоза яичников // Проблемы эндокринологии. - 2020. - Т. 66. - №6. - С. 74-80. doi: https://doi.org/10.14341/probl12620

\section{TO CITE THIS ARTICLE:}

levleva KD, Danusevich IN, Suturina LV. Role of leptin and nuclear receptor PPARY in PCOS pathogenesis. Problems of Endocrinology. 2020;66(6):74-80. doi: https://doi.org/10.14341/probl12620 